\begin{tabular}{|c|l|}
\hline Title & A nomaly of fractal dimensions observed in stochastically switched systems \\
\hline Author(s) & Nishikawa, Jun; Gohara, Kazutoshi \\
\hline Citation & $\begin{array}{l}\text { Physical Review E, 77(3), 036210_1-036210-8 } \\
\text { https://doi.org/L0.1103/PhysRevE.77.036210 }\end{array}$ \\
\hline Issue Date & 2008-03 \\
\hline Doc URL & http://hdl.handle.net/2115/45417 \\
\hline Rights & ○ 2008The American Physical Society \\
\hline Type & article \\
\hline File Information & 20080318Nishikawa_PRE.pdf \\
\hline
\end{tabular}

Instructions for use 


\title{
Anomaly of fractal dimensions observed in stochastically switched systems
}

\author{
Jun Nishikawa ${ }^{1,2}$ and Kazutoshi Gohara ${ }^{1}$ \\ ${ }^{1}$ Department of Applied Physics, Hokkaido University, Sapporo, Hokkaido 060-8628, Japan \\ ${ }^{2}$ RIKEN Brain Science Institute (BSI), Wako, Saitama 351-0198, Japan
}

(Received 24 October 2007; revised manuscript received 24 January 2008; published 18 March 2008)

\begin{abstract}
We studied an anomaly in fractal dimensions measured from the attractors of dynamical systems driven by stochastically switched inputs. We calculated the dimensions for different switching time lengths in twodimensional linear dynamical systems, and found that changes in the dimensions due to the switching time length had a singular point when the system matrix had two different real eigenvalues. Using partial dimensions along each eigenvector, we explicitly derived a generalized dimension $D_{q}$ and a multifractal spectrum $f(\alpha)$ to explain this anomalous property. The results from numerical calculations agreed well with those from analytical equations. We found that this anomaly is caused by linear independence, inhomogeneity of eigenvalues, and overlapping conditions. The mechanism for the anomaly could be identified for various inhomogeneous systems including nonlinear ones, and this reminded us of anomalies in some physical values observed in critical phenomena.
\end{abstract}

DOI: 10.1103/PhysRevE.77.036210

PACS number(s): 05.45.Df, 64.60.A-, 64.60.Cn

\section{INTRODUCTION}

Fractals observed in various physical phenomena have enthusiastically been studied, and examples of these have been in surface growth [1], colloids [2], optical systems [3], and turbulence [4]. Various models, which describe the behavior of systems, have also been presented and the fractals obtained have been analyzed using fractal dimensions [5-9]. These intensive studies have verified that the fractal dimension is one of the most important properties of fractals. Fractal dimensions were expanded to a generalized dimension $D_{q}$ and a multifractal spectrum $f(\alpha)$ to represent multiple fractal properties in different regions [10,11]. This multifractal formalism gave us a tool for analyzing various physical phenomena in the real world [12]. Therefore, from a theoretical viewpoint, it is important to derive $D_{q}$ and $f(\alpha)$ explicitly in objective systems.

For the fractals generated by the iterated function system (IFS), we can analytically derive $D_{q}$ and $f(\alpha)$ if each iterated function is a contraction map and the fractal set is totally disconnected set $[13,14]$. This conventional theory well describes fractal dimensions in various situations [15]. However, we could not derive them if these conditions are not valid. In the present study, we discuss anomalous property of fractal dimensions in such an invalid situation for the conventional theory.

IFS based on maps often suffer from difficulties to apply it to continuous systems. To overcome this point, one of the authors has presented a theoretical framework for a continuous dynamical system stochastically excited by external temporal inputs. It has been revealed that this dynamics is characterized by fractal trajectory $\Gamma(C)$ in hypercylindrical phase space $\mathcal{M}$ and fractal mapped point set $C$ on the Poincaré section $\Sigma[16,17]$. These fractals generated by switching vector fields have been observed in different domains such as a forced damped oscillator [18], human behavior [19], artificial neural networks [20], an electronic circuit [21], metal nanoparticles [22], and some physical situations [23]. This formalism could help us to connect IFS theory into more vari- ous physical situations described by ordinary differential equations.

In this paper, we present an anomaly observed in the fractal dimensions of the mapped point set $C$ generated by a continuous dynamical system with stochastically switched inputs. First, we analytically derive $D_{q}$ and $f(\alpha)$ based on the assumption of a single contraction factor, and calculate the fractal dimensions of the point sets generated by a twodimensional linear dynamical system to find the anomaly in the fractal dimensions. Next, we provide an explanation to enable this anomaly to be understood using a partial dimension method. Finally, we summarize our findings and draw conclusions.

\section{ANOMALY OF FRACTAL DIMENSIONS}

\section{A. Fractal transitions and fractal dimensions}

A dissipative nonautonomous dynamical system defined by the following ordinary differential equation is often used as a model of physical phenomena:

$$
\begin{gathered}
\dot{\boldsymbol{x}}=\boldsymbol{f}(\boldsymbol{x})+\boldsymbol{I}(t), \\
\boldsymbol{x}, \boldsymbol{f}, \boldsymbol{I} \in \boldsymbol{R}^{N},
\end{gathered}
$$

where $\boldsymbol{x}$ is the state, $\boldsymbol{f}$ is vector field, $\boldsymbol{I}$ is input, and $t$ is time. Equation (1) implies that a system characterized by state $\boldsymbol{x}$ is driven by an environment through temporal input $\boldsymbol{I}(t)$. In order to apply the theory to a variety of physical situations, we define the input as the stochastically switched sequence of plural temporal inputs $\boldsymbol{I}_{l}(t)$ with corresponding probability $p_{l}$ [16]. A set of the inputs can be expressed by $\left\{\boldsymbol{I}_{l}\right\}_{l=1}^{L}$. Here, $\boldsymbol{I}_{l}$ is the $l$ th input, and $L$ is the total number of inputs.

We can define two sets of dynamical systems corresponding to the set $\left\{\boldsymbol{I}_{l}\right\}$. The first is the set of continuous dynamical systems that is defined by the set $\left\{f_{l}\right\}$ of vector fields in hypercylindrical space $\mathcal{M}$, while the second is the set of discrete dynamical systems that is defined by the set $\left\{\boldsymbol{g}_{l}\right\}$ of iterated functions on the global Poincaré section $\Sigma$. We can 
obtain $\boldsymbol{g}_{l}$ by transformation from the continuous state variable $\boldsymbol{x}$ in Eq. (1) into a discrete variable $\boldsymbol{x}_{\tau}$ on the global Poincaré section $\Sigma$. When map $\boldsymbol{g}_{l}$ is a contraction, state $\boldsymbol{x}_{\tau}$ on the Poincaré section changes on the attractive and unique invariant set $C$ with a fractal structure after sufficient random iterations. Set $C$ satisfies the following equation $[13,14]$ :

$$
C=\stackrel{L}{\cup} g_{l}(C) .
$$

The fractal structure of set $C$ affects the corresponding trajectories. In cylindrical space $\mathcal{M}$, trajectory set $\Gamma(C)$ starting from initial set $C$ is obtained by the union of the trajectory set $\gamma_{l}(C)$ for each input $I_{l}[16]$,

$$
\Gamma(C)=\stackrel{L}{\cup} \gamma_{l=1}(C) .
$$

$\Gamma(C)$ is also an attractive and unique invariant set with a fractal-like structure. Here, we define an excited attractor as the final trajectory driven repeatedly by each input, and we designated this transition between each excited attractor as fractal transition.

Here, let us define a contraction factor $s_{l}$ of the iterated function $\boldsymbol{g}_{l}$ for two arbitrary points $\boldsymbol{x}_{1} \in \boldsymbol{R}^{N}$ and $\boldsymbol{x}_{2} \in \boldsymbol{R}^{N}$ on the Poincaré section as

$$
s_{l}\left(x_{1}, x_{2}\right)=\frac{\left|g_{l}\left(x_{1}\right)-g_{l}\left(x_{2}\right)\right|}{\left|x_{1}-x_{2}\right|} .
$$

If contraction factor $s_{l}$ is a constant of less than 1 in each input, $C$ becomes a fractal set. In this condition, we can derive generalized dimension $D_{q}$ of fractal set $C$ constructed by $L$ kind of inputs as in the following equation [11]:

$$
\sum_{l=1}^{L} p_{l}^{q} s_{l}^{(1-q) D_{q}}=1
$$

where $p_{l}$ and $s_{l}$ are the probability and contraction factor of the $l$ th input. Note that the results do not assume linearity of the function $f$. However, if contraction factor $s_{l}$ is not a constant, we cannot derive generalized dimension $D_{q}$ of fractal set $C$ even in the case of linear systems. In Sec. II C, we will demonstrate the anomaly in fractal dimensions in this type of condition.

\section{B. Fractal transitions in linear systems}

Here, we consider a following nonautonomous twodimensional linear dynamical system with switching inputs to demonstrate the fractal transition discussed in the preceding section:

$$
\dot{\boldsymbol{x}}=\boldsymbol{A} \boldsymbol{x}+\boldsymbol{I}_{l}(t),
$$

where

$$
\boldsymbol{x}=\left(\begin{array}{l}
x_{1} \\
x_{2}
\end{array}\right), \quad \boldsymbol{A}=\left(\begin{array}{ll}
a_{1} & a_{2} \\
a_{3} & a_{4}
\end{array}\right), \quad \boldsymbol{I}_{l}(t)=\left(\begin{array}{l}
I_{1, l}(t) \\
I_{2, l}(t)
\end{array}\right) .
$$

We first define a new variable $\theta=\left(2 \pi / T_{l}\right) t(\bmod 2 \pi)$, where $T_{l}$ is the duration of $l$ th input. $T_{l}$ can be considered as the switching time length because each input is stochastically switched one after another in this duration. Replacing variables $\left(x_{1}, x_{2}, \theta\right)$ with $\boldsymbol{y} \in \boldsymbol{R}^{2} \times \boldsymbol{S}^{1}$, i.e., cylindrical space $\mathcal{M}$, the nonautonomous system in Eq. (6) can be transformed into the following autonomous system:

$$
\dot{y}=f_{l}(\boldsymbol{y})=\left\{\begin{array}{l}
a_{1} x_{1}+a_{2} x_{2}+I_{1, l}\left(T_{l} \theta / 2 \pi\right), \\
a_{3} x_{1}+a_{4} x_{2}+I_{2, l}\left(T_{l} \theta / 2 \pi\right), \\
2 \pi / T_{l} .
\end{array}\right.
$$

Using new variables $\left(x_{1, \tau}, x_{2, \tau}\right) \equiv \boldsymbol{x}_{\tau} \in R^{2}$, which is Poincaré section $\Sigma$ at $\theta=0$ or $2 \pi$, we obtain the corresponding Poincaré mapping,

$$
\boldsymbol{x}_{\tau+1}=\boldsymbol{g}_{l}\left(\boldsymbol{x}_{\tau}\right)=\boldsymbol{A}_{l} \boldsymbol{x}_{\tau}+\boldsymbol{B}_{l},
$$

where

$$
\boldsymbol{A}_{l}=e^{\boldsymbol{A} T_{l}}, \quad \boldsymbol{B}_{l}=\int_{0}^{T_{l}} e^{-\boldsymbol{A}\left(s-T_{l}\right)} \boldsymbol{I}_{l}(s) d s
$$

The $2 \times 2$ matrix $\boldsymbol{A}$ represents the following three types of Jordan normal forms:

$$
\text { (I) }\left(\begin{array}{cc}
\lambda_{1} & 0 \\
0 & \lambda_{2}
\end{array}\right), \quad \text { (II) }\left(\begin{array}{cc}
\lambda & 0 \\
1 & \lambda
\end{array}\right), \quad \text { (III) }\left(\begin{array}{cc}
\alpha & -\beta \\
\beta & \alpha
\end{array}\right) .
$$

The matrices (I), (II), and (III) correspond to diagonalizable matrices, nondiagonalizable matrices, and matrices with complex eigenvalues. Here, we derive a contraction factor for each case using the Wada and Gohara method [24]. The contraction factor $s_{l}\left(\boldsymbol{x}_{1}, \boldsymbol{x}_{2}\right)$ of iterated function $\boldsymbol{g}_{l}$ is transformed from Eq. (4) into

$$
s\left(\boldsymbol{x}_{1}, \boldsymbol{x}_{2}\right)=\frac{\left|e^{\boldsymbol{A} T_{l}} \boldsymbol{x}_{1}+\boldsymbol{B}_{l}-\left(e^{\boldsymbol{A} T_{l}} \boldsymbol{x}_{2}+\boldsymbol{B}_{l}\right)\right|}{\left|\boldsymbol{x}_{1}-\boldsymbol{x}_{2}\right|}=\frac{\mid e^{\boldsymbol{A} T_{l}\left(\boldsymbol{x}_{1}-\boldsymbol{x}_{2}\right) \mid}}{\left|\boldsymbol{x}_{1}-\boldsymbol{x}_{2}\right|},
$$

where $\boldsymbol{x}_{1}$ and $\boldsymbol{x}_{2}$ are two arbitrary points on the plane $\boldsymbol{R}^{2}$. Term $\boldsymbol{B}_{l}$ is eliminated and does not influence the contraction factor. Let $e^{A T_{l}} \equiv\left(\begin{array}{ll}a & b \\ c & d\end{array}\right)$ and let $z_{j(j=1,2)}$ be the $j$ th component of the difference vector $\boldsymbol{x}_{1}-\boldsymbol{x}_{2}$. If $z_{1} \neq 0$, the contraction factor in Eq. (10) can be rewritten as

$$
\begin{aligned}
s\left(\boldsymbol{x}_{1}, \boldsymbol{x}_{2}\right) & =\frac{\sqrt{\left(a z_{1}+b z_{2}\right)^{2}+\left(c z_{1}+d z_{2}\right)^{2}}}{\sqrt{z_{1}^{2}+z_{2}^{2}}} \\
& =\frac{\sqrt{\left(a^{2}+c^{2}\right)+2(a b+c d) r+\left(b^{2}+d^{2}\right) r^{2}}}{\sqrt{1+r^{2}}},
\end{aligned}
$$

where $r$ is the ratio $\frac{z_{2}}{z_{1}}$. Equation (11) shows that the contraction factor only depends on variable $r$, and can be denoted by $s(r)$. If $z_{1}=0$, the contraction factor is given by $\sqrt{\left(b^{2}+d^{2}\right)}$, which is equal to $\lim _{r \rightarrow \pm \infty} s(r)$. Using all matrices $\boldsymbol{A}_{l}$ of all iterated functions, 


$$
\begin{aligned}
& \text { (I) }\left(\begin{array}{cc}
e^{\lambda_{1} T_{l}} & 0 \\
0 & e^{\lambda_{2} T_{l}}
\end{array}\right), \quad \text { (II) }\left(\begin{array}{cc}
e^{\lambda T_{l}} & 0 \\
T_{l} e^{\lambda T_{l}} & e^{\lambda T_{l}}
\end{array}\right), \\
& \text { (III) }\left(\begin{array}{cc}
e^{\alpha T_{l}} \cos \beta T_{l} & -e^{\alpha T_{l}} \sin \beta T_{l} \\
e^{\alpha T_{l}} \sin \beta T_{l} & e^{\alpha T_{l}} \cos \beta T_{l}
\end{array}\right),
\end{aligned}
$$

we can analytically calculate the contraction factor using Eqs. (11) and (12). For matrix (I), $S(r)$ is expressed as

$$
s(r)=\sqrt{\frac{e^{2 \lambda_{1} T_{l}}+e^{2 \lambda_{2} T_{l} r^{2}}}{1+r^{2}}} .
$$

If the eigenvalues satisfy $\lambda_{1}=\lambda_{2} \equiv \lambda$, contraction factor $s(r)$ becomes a constant $e^{\lambda T_{l}}$. However, for the eigenvalues $\lambda_{1}$ $\neq \lambda_{2}, s(r)$ does not become a constant. For matrix (II), $S(r)$ is expressed as

$$
s(r)=e^{\lambda T_{l}} \sqrt{1+T_{l}^{2}\left(\frac{1+\frac{2 r}{T_{l}}}{1+r^{2}}\right)} .
$$

The contraction factor is not a constant in this case, but converges to constant $e^{\lambda T_{l}}$ on a sufficiently smaller scale of point set $C$. We will discuss the reason the contraction factor converges into a constant in Appendix B. For matrix (III), $S(r)$ is expressed as

$$
s(r)=e^{\alpha T_{l}} .
$$

If the real part of the eigenvalue satisfies $\alpha \equiv \lambda$, contraction factor $s(r)$ becomes a constant $e^{\lambda T_{l}}$.

For matrix (I) with $\lambda_{1}=\lambda_{2} \equiv \lambda$, matrices (II), and matrices (III) with $\alpha \equiv \lambda$, we can derive generalized dimension $D_{q}$ of fractal set $C$ constructed with $L$ types of inputs using Eq. (5) because contraction factor $s_{l}$ is constant under this condition. When $T_{l}=T \forall l, D_{q}$ is explicitly obtained as

$$
D_{q}=\frac{1}{q-1} \frac{\ln \left(\sum_{l=1}^{L} p_{l}^{q}\right)}{\lambda T} .
$$

Using Legendre transformation we can obtain $\alpha$ and $f(\alpha)$ as follows $[10,11]$ :

$$
\begin{gathered}
\alpha=\frac{\sum_{l=1}^{L}\left(p_{l}^{q} \ln p_{l}\right)}{\lambda T \sum_{l=1}^{L} p_{l}^{q}}, \\
f(\alpha)=\frac{q \sum_{l=1}^{L}\left(p_{l}^{q} \ln p_{l}\right)}{\lambda T \sum_{l=1}^{L} p_{l}^{q}}-\frac{\ln \left(\sum_{l=1}^{L} p_{l}^{q}\right)}{\lambda T} .
\end{gathered}
$$

\section{Anomaly of fractal dimensions}

Unlike the case of single contraction factor in the preceding section, we cannot derive generalized dimension $D_{q}$ and multifractal spectrum $f(\alpha)$ for matrix (I) with $\lambda_{1} \neq \lambda_{2}$ because the contraction factor is not a constant in this case. This situation is common to a wide range of inhomogeneous systems. We will present the results of a numerical experiment in such a system, and demonstrate an anomaly of the dimension. We will present the result for the correlation dimension $D_{2}$, which is $D_{q}$ for $q=2$. Although we have only presented the result for $D_{2}$ for simplicity, we have obtained the same results for all $q$. In the following examples, we assumed that two inputs $\boldsymbol{I}_{1}=(1.0,1.0)$ and $\boldsymbol{I}_{2}=(-1.0,-1.0)$ were stochastically switched with probability $p_{1}=0.7, p_{2}$ $=0.3$, one after another, while the switching time length was $T_{1}=T, T_{2}=T$.

First, let us examine system matrix (I) with homogeneous eigenvalues $\lambda=\lambda_{1}=\lambda_{2}=-0.5$ as an example of a trivial case, in which the fractal dimension obeys conventional theory. If input $\boldsymbol{I}_{1}$ is fed into the system repeatedly, the system converges into a corresponding excited attractor $\boldsymbol{A t t}_{1}=(2.0,2.0)$. In contrast, if input $\boldsymbol{I}_{2}$ is fed into the system repeatedly, the system converges into a corresponding excited attractor $\boldsymbol{A t t}_{2}=(-2.0,-2.0)$. When the input is stochastically switched, the system converges into an attractor with a fractal structure around each excited attractor and we can observe fractal set $C$ on the Poincaré section. Here, we calculated correlation dimension $D_{2}$ of fractal set $C$ according to changing input switching time lengths $T$ from 0.1 to 5.0. The bottom graph in Fig. 1 shows calculated $D_{2}$ of set $C$ indicated by the crosses "+" at all switching time lengths $T$, and the top panels in the figure show fractal sets $C$ on the Poincare section for time lengths $T$ of 0.5, 1.3, 1.8, 3.0, and 5.0. Each arrow from each panel points to a corresponding cross + in the bottom graph. The theoretical curve derived from Eq. (16) is denoted by the dotted curve in the graph. We can intuitively understand the extreme situations in both sides. For $T \rightarrow \infty$, the point sets converge into the two excited attractors because the system has sufficient time to converge all excited attractors before switching. This situation can be seen in Fig. 1(e). Consequently, the correlation dimension converges into 0 according to the theoretical curve. On the other hand, for $T \rightarrow 0$, the system has no time to converge the attractors before switching. Therefore, the point sets cannot be divided into totally disconnected clusters, but they overlapped with each other. In addition, the homogeneous contraction factor leads the point sets to spread along a single direction. This situation makes the point set to be a line in the twodimensional (2D) state space as shown in Fig. 1(a). As a result, the correlation dimension converges into 1 . We can analytically derive the critical time length from the intersection between $D_{2}=1$ and Eq. (16). In this case, the critical time length $T \simeq 1.09$. In the intermediate value of $T$, the theoretical curve fits the result of the numerical experiment well except for the overlapping situation in $T<1.09$. This is consistent with the theory introduced previously.

Now, we present the results for a nontrivial case with inhomogeneous eigenvalues. In this condition, the contraction factor is not a constant and varies with directions. Therefore, we cannot derive the fractal dimension by the conventional theory. We present the case for matrix (I) with inhomogeneous eigenvalues $\lambda_{1}=-5.0$ and $\lambda_{2}=-0.5$. Each excited attractor is $\boldsymbol{A t t} t_{1}=(0.2,2.0)$ and $\boldsymbol{A t t}_{2}=(-0.2,-2.0)$. Simi- 
(a) $T=0.5$
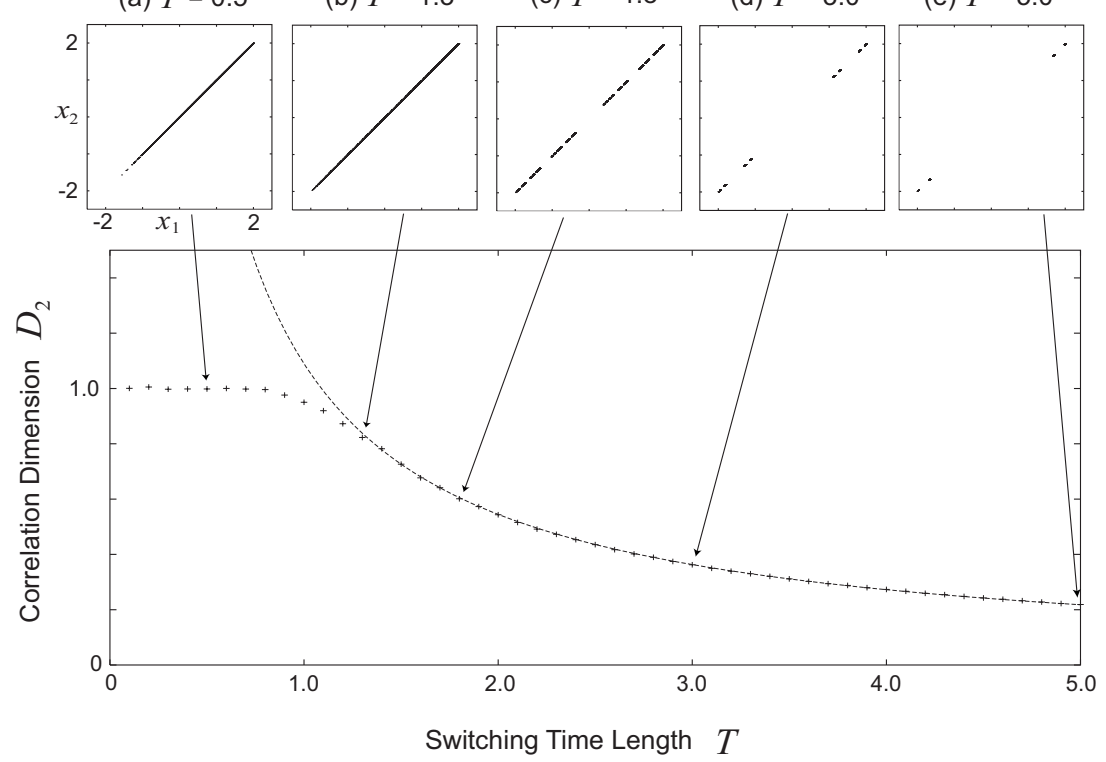

FIG. 1. Correlation dimension $D_{2}$ to switching time length $T_{l}$ of inputs for matrix (I) with homogeneous eigenvalues. Crosses $(+)$ indicate dimensions computed from fractal set $C$ on Poincaré section $\Sigma$, while the dotted curve indicates the theoretical curve of Eq. (16). (a), (b), (c), (d), and (e) in the top panels indicate point sets of fractal $C$ in input time lengths of $0.5,1.3,1.8,3.0$, and 5.0. larly, we calculated correlation dimension $D_{2}$ for switching time lengths $T$ from 0.1 to 5.0. The bottom graph in Fig. 2 plots the calculated $D_{2}$ indicated by the crosses "+" at all switching time lengths $T$, and the top panels in the figure show fractal sets $C$ on the Poincaré section for time lengths $T$ of $0.3,0.6,1.0,1.5$, and 5.0. For $T \rightarrow 0$, the point sets spread along the plane because of rapid switching and the inhomogeneous contraction factor. As a result, the correlation dimension converges into 2 . However, for $T \rightarrow \infty$, the point sets converges into the two excited attractors because the system has sufficient time to converge all excited attractors before switching. However, we cannot understand the curious changes in the dimensions between these two limits. The correlation dimensions do not fit into a theoretical curve according to Eq. (16), but they fit well with curve $B$ in the region of short $T(T<0.9)$, and fit well with curve $A$ in the region of long $T(T>1.2)$. There is a singular point around
$T \simeq 1.15$ for the dimensions. This implies that the properties of fractal dynamics change discontinuously among the singular point. What mechanism underlies this change in the dimensions? We will explain the origin of this anomaly, and derive two theoretical curves (curves $A$ and $B$ ) in the following section.

\section{ORIGIN OF ANOMALY}

This section presents the origin of the anomaly observed for inhomogeneous eigenvalues using the partial-dimension method [25]. We can define generalized dimension $D_{q}$ as the sum of all partial dimensions $D_{q, 1}, D_{q, 2}$ as

$$
D_{q}=D_{q, 1}+D_{q, 2}
$$

All dimensions satisfy the following equation:

(e) $T=5.0$

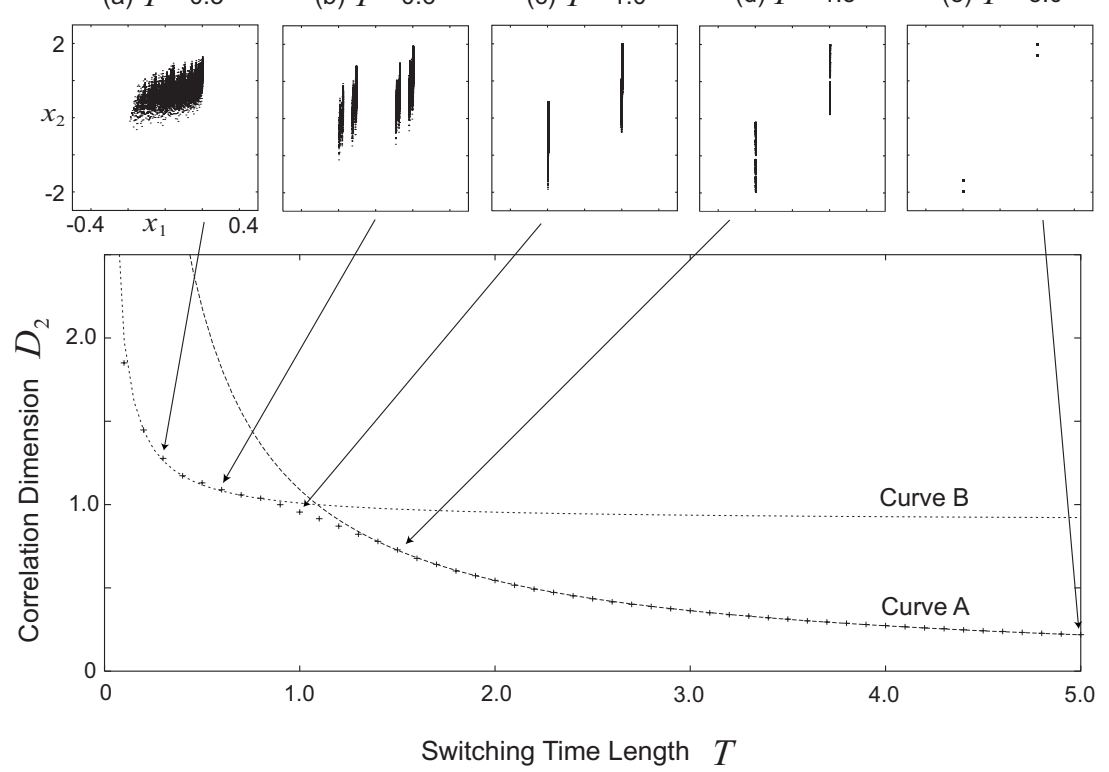

FIG. 2. Correlation dimension $D_{2}$ to switching time length $T_{l}$ of inputs for matrix (I) with heterogeneous eigenvalues. Curves $A$ and $B$ indicate theoretical curves discussed in Sec. III. Curve $A$ satisfies Eq. (23), and curve $B$ satisfies Eq. (28). 


$$
\sum_{l=1}^{L} p_{l}^{q} e^{\lambda_{1} T_{l}(1-q) D_{q, 1}} e^{\lambda_{2} T_{l}(1-q) D_{q, 2}}=1,
$$

where two eigenvalues have the relation $\lambda_{1}<\lambda_{2}$, as in the example in the preceding section. In a sufficiently large scale $\left(T>T_{c}\right)$, the fractal set converges to a point along the eigenvector with eigenvalue $\lambda_{1}$, and partial dimension $D_{q, 1}$ approximately converges to zero as

$$
D_{q, 1} \simeq 0 \text {. }
$$

Therefore, in this case, we can derive

$$
\sum_{l=1}^{L} p_{l}^{q} e^{\lambda_{2} T_{l}(1-q) D_{q}}=1 .
$$

When $T_{l}=T \forall l, D_{q}$ is explicitly obtained as

$$
D_{q}=\frac{1}{q-1} \frac{\ln \left(\sum_{l=1}^{L} p_{l}^{q}\right)}{\lambda_{2} T} .
$$

Curve $A$ in Fig. 2 is plotted according to Eq. (23) with $q$ $=2$. Consistent with the assumption in Eq. (21), calculated correlation dimension $D_{2}$ fits the theoretical curve well with larger $T$. Using Legendre transformation, we can also obtain $\alpha$ and $f(\alpha)$ as

$$
\begin{gathered}
\alpha=\frac{\sum_{l=1}^{L}\left(p_{l}^{q} \ln p_{l}\right)}{\lambda_{2} T \sum_{l=1}^{L} p_{l}^{q}} \\
f(\alpha)=\frac{q \sum_{l=1}^{L}\left(p_{l}^{q} \ln p_{l}\right) \ln \left(\sum_{l=1}^{L} p_{l}^{q}\right)}{\lambda_{2} T} . \\
\lambda_{2} T \sum_{l=1}^{L} p_{l}^{q}
\end{gathered}
$$

However, for $T \leq T_{c}$, clusters of the fractal set overlap along the eigenvector with eigenvalue $\lambda_{2}$ because of rapid switching. As a result, partial dimension $D_{q, 2}$ converges to one as

$$
D_{q, 2} \simeq 1 \text {. }
$$

Therefore, in this case, we can derive

$$
\sum_{l=1}^{L} p_{l}^{q} e^{\lambda_{1} T_{l}(1-q)\left(D_{q}-1\right)} e^{\lambda_{2} T_{l}(1-q)}=1 .
$$

When $T_{l}=T \forall l, D_{q}$ is explicitly obtained as

$$
D_{q}=\frac{1}{q-1} \frac{\ln \left(\sum_{l=1}^{L} p_{l}^{q}\right)}{\lambda_{1} T}+1-\frac{\lambda_{2}}{\lambda_{1}} .
$$

Curve $B$ in Fig. 2 is plotted according to Eq. (28) with $q$ $=2$. Consistent with the assumption in Eq. (26), calculated correlation dimension $D_{2}$ fits the theoretical curve well with shorter $T$. Using Legendre transformation, we can also obtain $\alpha$ and $f(\alpha)$,

$$
\alpha=\frac{\sum_{l=1}^{L}\left(p_{l}^{q} \ln p_{l}\right)}{\lambda_{1} T \sum_{l=1}^{L} p_{l}^{q}}+1-\frac{\lambda_{2}}{\lambda_{1}}
$$

$$
f(\alpha)=\frac{q \sum_{l=1}^{L}\left(p_{l}^{q} \ln p_{l}\right)}{\lambda_{1} T \sum_{l=1}^{L} p_{l}^{q}}-\frac{\ln \left(\sum_{l=1}^{L} p_{l}^{q}\right)}{\lambda_{1} T}+1-\frac{\lambda_{2}}{\lambda_{1}} .
$$

The critical time length $T_{c}$ is the point when partial dimension $D_{q, 2}$ is overlapped. Therefore, we can derive $T_{c}$ from Eq. (23) under condition $D_{q, 2}=D_{q}=1$ as

$$
T_{c}=\frac{\ln \left(\sum_{l=1}^{L} p_{l}^{q}\right)}{\lambda_{2}(q-1)} .
$$

In the case of inhomogeneous matrix (I) as discussed in the preceding section, the critical time length is calculated as $T_{c} \simeq 1.1563699 \ldots$, which is the cross point for curves $A$ and $B$ in Fig. 2 .

We found that this anomaly is caused by linear independence, inhomogeneity of eigenvalues, and overlapping conditions. Because of the linear independence between all eigenvectors, we can divide the fractal dimension into partial dimensions. Because of the inhomogeneity of eigenvalues, the fractal set is compressed in one direction for $T>T_{c}$, and the dimension is only determined by the eigenvalue in the other direction as Eq. (23). However, for $T<T_{c}$, the fractal set overlaps, and is not sufficiently compressed. As a result, the dimension is determined by both eigenvalues as Eq. (28).

\section{SUMMARY AND DISCUSSION}

We studied an anomaly in fractal dimensions calculated from the attractors of stochastically switched continuous dynamical systems. As a result of calculating a twodimensional linear dynamical system with two inputs, we found that there was a singular point in the change of fractal dimensions at the switching time length $T$ when there were different contractions along directions. We verified the origin of this anomaly using a partial-dimension method, and explicitly derived a generalized dimension $D_{q}$ and a multifractal spectrum $f(\alpha)$. This anomaly is caused by linear independence, inhomogeneity of eigenvalues, and overlapping conditions of the fractal set. Although we demonstrated the anomalous property of fractal dimensions in a simple twodimensional dynamical system, the obtained mechanism for the anomaly could be identified for various inhomogeneous systems including nonlinear ones, and this reminded us of anomalies in some physical values observed in critical phenomena. We conjectured that overlapping conditions of inho- 
mogeneous systems corresponds to symmetry breaking in various physical situations.

Although the fractal dimension is one of the most important properties of fractals, it is often difficult to derive it analytically. However, it has been known that we can derive the dimension of fractal set generated by IFS in a certain condition $[13,14]$. In this formalism, each iterated function must be a contraction map. In addition, the fractal set must be a totally disconnected set, in other words, it must not be an overlapping condition. If the objected systems or models do not satisfy these conditions, we cannot derive the dimension explicitly. In the present study, we demonstrated anomaly of fractal dimension caused by the situation that the timing of overlap is different in each direction. Using partialdimension method, we analytically derived the fractal dimension that can explain this anomalous property. These results will contribute the IFS theory to be a more sophisticated form, and they could be applied to critical phenomena or phase transition in the relation with fractal dimensions.

IFS is defined by the set of iterated functions, each of which is the contraction maps. This property often makes it difficult to apply it to continuous time systems. The framework for a continuous dynamical system stochastically excited by external temporal inputs $[16,17]$ is useful to discuss the properties of fractal dimensions in continuous systems. One of the strong points in this framework is that we can simultaneously describe the switched continuous system and the corresponding iterated function system in this framework. This could help us to apply IFS theory to more various physical situations described by ordinary differential equations [18-23]. Therefore, we also use this framework in the present study to demonstrate the anomaly of fractal dimensions. As a result, the obtained theoretical equations have a parameter (switching time length $T$ ), which is found only in continuous systems. This result could not be obtained if we only start from conventional iterated function systems based on maps.
There has been one study that demonstrates phase transitions in a chaotically modulated linear system [26]. This study presented a jump of the fractal dimension from one theoretical curve to another as similar as the present study. As a result of a rigorous analysis of the fractal dimension according to the thermodynamic formalism of dynamical systems, they verified that this transition comes from the competition between the two contracting directions. This is completely the same as the origin of the present study. On the contrary, there are many differences between them. First, they did not discuss the overlapping condition in each direction, which has a very important role in the present study. Second, they did not mention about the relation to IFS theory. Third, presented models are qualitatively different between them. They used the filtered Baker map as an example of a nontrivial hyperbolic three-dimensional map, which is a completely deterministic but chaotic system. We used a 2D linear dynamical system driven by stochastically switched inputs. The relation between these two models could be generalized into the relation between strange attractors of a chaotic system and fractal sets generated by IFS. To clear this point might be the next step toward the understanding of chaos and fractals.

\section{ACKNOWLEDGMENT}

We thank Jun-ichiro Okuno for his valuable advice in the analysis of partial-dimension method and preliminary calculation of fractal dimensions.

\section{APPENDIX A: RESULTS FOR SINGLE CONTRACTION FACTOR}

This appendix explains the change in correlation dimension $D_{2}$ to switching time length $T$ for a single contraction factor.

First, let us present the case of system matrix (II) with $\lambda=-0.5$. Each excited attractor is $\boldsymbol{A} t t_{1}=(2.0,6.0)$ and $\boldsymbol{A} t t_{2}$

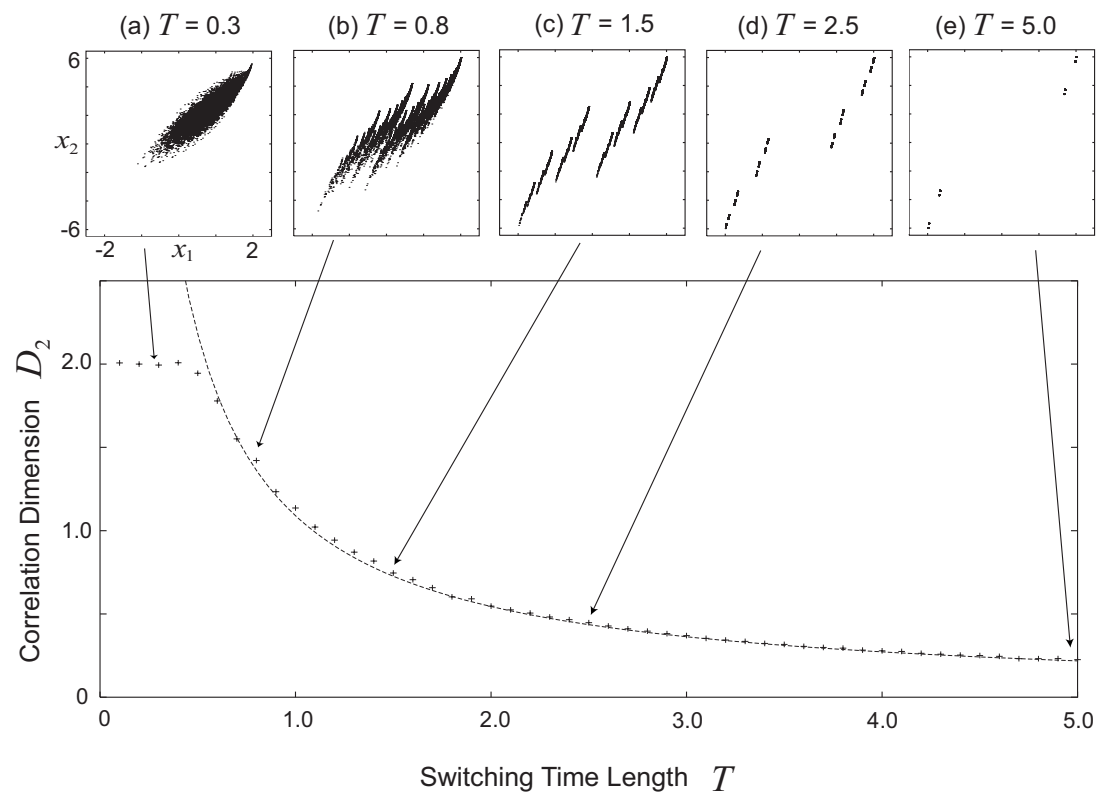

FIG. 3. Correlation dimension $D_{2}$ to switching time length $T_{l}$ of inputs for matrix (II). 
(a) $T=0.3$

(b) $T=0.6$

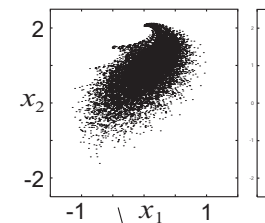

(c) $T=0.8$

(d) $T=1.5$

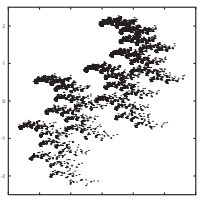

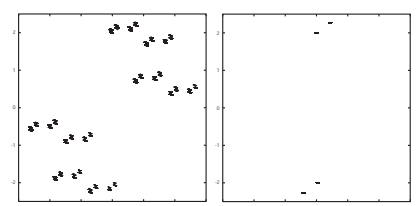

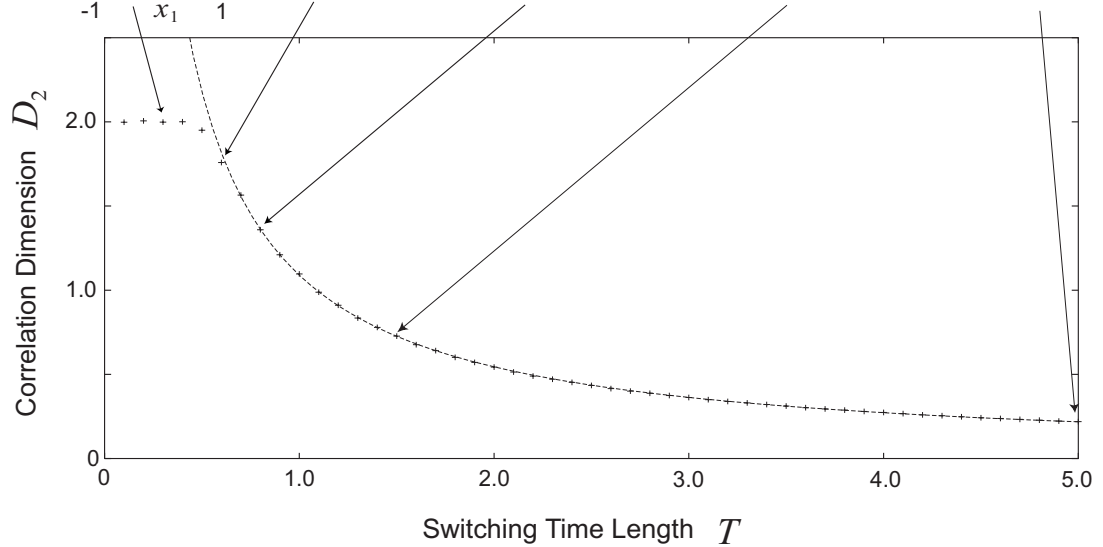

FIG. 4. Correlation dimension $\mathrm{D}_{2}$ to switching time length $T_{l}$ of inputs for matrix (III).

$=(-2.0,-6.0)$. Similarly, we calculated correlation dimension $D_{2}$ for switching time lengths $T$ from 0.1 to 5.0. The bottom graph in Fig. 3 plots the calculated $D_{2}$, indicated by the crosses, at all input time lengths $T$, and the top panels in the figure show the fractal sets $C$ on the Poincaré section for time lengths of $T=0.3,0.8,1.5,2.5$, and 5.0. The theoretical curve is also plotted according to Eq. (16), and the results fit well except for some systematic upper shift. In Appendix B, we will discuss the origin of this upper shift; it disappears as the number of points is increased and smaller scale data are used.

Second, let us present the case of the system matrix (III) with $\alpha=-0.5$ and $\beta=0.5$. Each excited attractor is $\boldsymbol{A t t}_{1}$

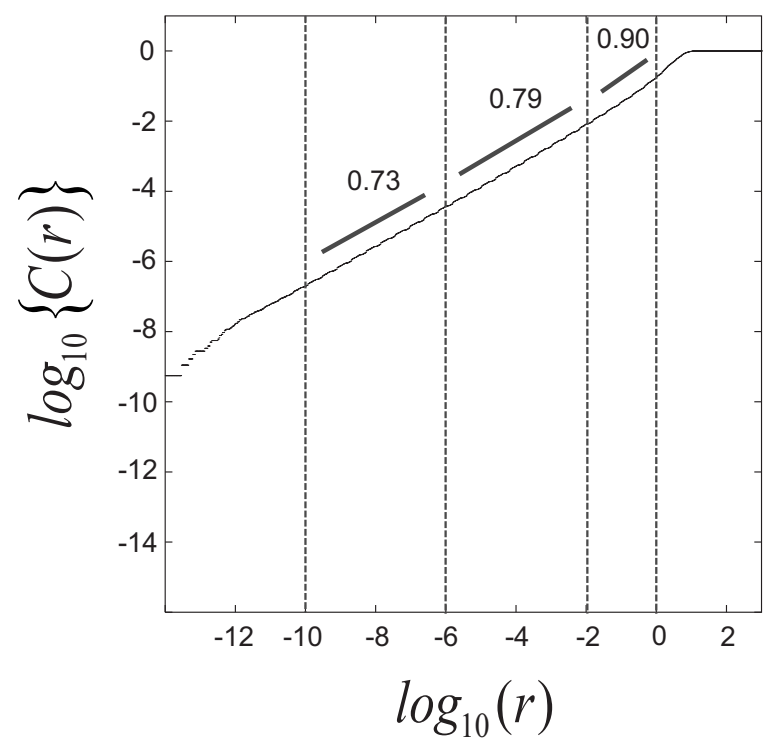

FIG. 5. Multiple scaling properties of fractal set $C$. The figure shows the relationship of logarithm of correlation integral $C(r)$ to logarithm of scale $r$. The base of logarithm is 10 in this figure. Calculated $D_{2}$ is 0.90 in the region from $-2 \leq r<0,0.79$ in -6 $\leq r<-2$, and 0.73 in $-10 \leq r<-6$.
$=(0.0,2.0)$ and $\boldsymbol{A t t}_{2}=(0.0,-2.0)$. Similarly, we calculated correlation dimension $D_{2}$ for switching time lengths $T$ from 0.1 to 5.0. The bottom graph in Fig. 4 plots the calculated $D_{2}$, indicated by the crosses at all input time lengths $T$, and the top panels in the figure show the fractal sets $C$ on the Poincaré section for time lengths of $T=0.3,0.6,0.8,1.5$, and 5.0. The theoretical curve is also plotted according to Eq. (16) with $\lambda=-0.5$, and the results fit well.

We can conclude that Eq. (16) worked well in all types of two-dimensional linear dynamical systems with single constant contraction factors from the results in Sec. II C and these two results for system matrix (II) and system matrix (III).

\section{APPENDIX B: MULTIPLE SCALING PROPERTIES}

Here, we discuss the multiple scaling properties observed in system matrix (II) presented in Appendix A.

Because the contraction factor $s_{l}$ does not depend on the second term $B_{l}$, discussed in Sec. II B, we will ignore the second term from now on. Consequently, we can obtain recurrence formulas from the discussion in Sec. II B as

$$
\begin{gathered}
x_{1, n}=e^{\lambda T_{l} x_{1, n-1},} \\
x_{2, n}=T e^{\lambda T_{l}} x_{2, n-1}+e^{\lambda T_{l}} x_{1, n-1},
\end{gathered}
$$

where $x_{i, n}$ is the $i$ th component of the $n$-times-iterated scale for the attractor. Using the initial scale, $x_{1,0}$ and $x_{2,0}$, we can derive the following relations:

$$
\begin{gathered}
x_{1, n}=e^{n \lambda T_{l}} x_{1,0}, \\
x_{2, n}=n T_{l} e^{n \lambda T_{l}} x_{2,0}+e^{n \lambda T_{l}} x_{1,0} .
\end{gathered}
$$

Here, the $x_{1}$ component of the contraction factor $s_{l}$ is easily obtained as $e^{\lambda T_{l}}$. However, the $x_{2}$ component of $e^{\lambda T_{l}}$ does not have a constant value and depends on the $x_{1}$ component. Under the condition of $n \rightarrow \infty$, the $x_{2}$ component of the con- 
traction factor converges into the same value as the $x_{1}$ component as

$$
\lim _{n \rightarrow \infty} \frac{x_{2, n}}{x_{2, n-1}}=\lim _{n \rightarrow \infty} \frac{n T_{l} e^{n \lambda T_{l}} x_{1,0}+e^{n \lambda T_{l}} x_{2,0}}{(n-1) T_{l} e^{(n-1) \lambda T_{l}} x_{1,0}+e^{(n-1) \lambda T_{l}} x_{2,0}}=e^{\lambda T_{l}} .
$$

This means that contraction factor $s_{l}$ converges into $e^{\lambda T_{l}}$ in all directions. Thus, we can approximate the dimension to Eq. (16) with eigenvalue $\lambda$. Actually, calculated correlation dimension $\mathrm{D}_{2}$ fits the theoretical curve well in Fig. 3. The small upper shift of $D_{2}$ seems to be caused by insufficient convergence in the contraction factor. This may be decreased by calculating the dimension using more small-scale data. An example of this effect is presented in Fig. 5. If we use the data from -2 to 0 to calculate the dimension, we obtain 0.90 . Similarly, we obtain 0.79 using the data from -6 to -2 , and 0.73 using the data from -10 to -6 . The theoretical value from Eq. (16) is about 0.73 . The results show that the value of the correlation dimension converges into the theoretical value according to the decrease in the scale of calculation. Note that we need to sample a sufficient number of points to evaluate it on a small scale. Such multiple scaling properties have been observed in some areas, and, these properties are thought to be important to analyze complex systems [27].
[1] R. M. Brady and R. C. Ball, Nature (London) 309, 225 (1984).

[2] D. W. Schaefer, J. E. Martin, P. Wiltzius, and D. S. Cannell, Phys. Rev. Lett. 52, 2371 (1984).

[3] F. T. Arecchi, W. Gadomski, and R. Meucci, Phys. Rev. A 34, 1617 (1986).

[4] J. C. Sommerer and E. Ott, Science 259, 335 (1993).

[5] T. A. Witten, Jr. and L. M. Sander, Phys. Rev. Lett. 47, 1400 (1981).

[6] R. C. Ball and E. Somfai, Phys. Rev. Lett. 89, 135503 (2002).

[7] B. Davidovitch, H. G. E. Hentschel, Z. Olami, I. Procaccia, L. M. Sander, and E. Somfai, Phys. Rev. E 59, 1368 (1999).

[8] E. Ott and T. M. Antonsen, Jr., Phys. Rev. Lett. 61, 2839 (1988).

[9] L. Yu, E. Ott, and Q. Chen, Phys. Rev. Lett. 65, 2935 (1990).

[10] H. G. E. Hentschel and I. Procaccia, Physica D 8, 435 (1983).

[11] T. C. Halsey, M. H. Jensen, L. P. Kadanoff, I. Procaccia, and B. I. Shraiman, Phys. Rev. A 33, 1141 (1986).

[12] M. H. Jensen, L. P. Kadanoff, A. Libchaber, I. Procaccia, and J. Stavans, Phys. Rev. Lett. 55, 2798 (1985).

[13] M. F. Barnsley, Fractals Everywhere, 2nd ed. (Academic, Boston, 1993).
[14] J. Hutchinson, Indiana Univ. Math. J. 30, 713 (1981).

[15] T. M. Antonsen, Jr., A. Namenson, E. Ott, and J. C. Sommerer, Phys. Rev. Lett. 75, 3438 (1995).

[16] K. Gohara and A. Okuyama, Fractals 7, 205 (1999).

[17] K. Gohara and A. Okuyama, Fractals 7, 313 (1999).

[18] K. Gohara, H. Sakurai, and S. Sato, Fractals 8, 67 (2000).

[19] Y. Yamamoto and K. Gohara, Hum. Mov. Sci. 19, 341 (2000).

[20] S. Sato and K. Gohara, Int. J. Bifurcation Chaos 11, 421 (2001).

[21] J. Nishikawa and K. Gohara, Int. J. Bifurcation Chaos 12, 827 (2002).

[22] N. Nishiguchi, Phys. Rev. Lett. 89, 066802 (2002).

[23] J. M. Kowalski, in Fractals and Beyond: Complexities in the Sciences, edited by M. M. Novak (World Scientific, Singapore, 1998).

[24] R. Wada and K. Gohara, Int. J. Bifurcation Chaos 11, 755 (2001).

[25] T. Morita et al., Prog. Theor. Phys. 79, 296 (1988).

[26] P. Paoli, A. Politi, and R. Badii, Physica D 36, 263 (1989).

[27] K. Chen and P. Bak, Phys. Rev. E 62, 1613 (2000). 